\title{
ACRN JOURNAL OF FINANCE AND RISK PERSPECTIVES
}

ISSN 2305-7394

VOLUME 11

2022

\section{CONTENTS}

Editorial Global Perspectives

Othmar Lehner

Risk Factors in the German Stock Market:

Can Sentiment Improve the Performance of Traditional Multifactor Models?

Emile David Hövel, Matthias Gehrke

Reconstruction of the Slippery Slope Framework Tax Compliance Model

I Nyoman Darmayasa, I Made Marsa Arsana, I Made Agus Putrayasa

The Impact of Real Manipulation and Tax Management on Future Market

Value: An Artificial Intelligence Simulation of High Earnings Quality

Muljanto Siladjaja, Yuli Anwar, Ismulyana Djan

Does transaction atmosphere influence the decision-making

behaviour of investors?

Christa Hangl, Matthias Ortner

A comparison of market risk measures from a twofold perspective: accurate and loss function

Sonia Benito Muela, Carmen López-Martin, Raquel Arguedas-Sanz

The role of forensic auditing techniques in preventing non-government organisations' financial statement fraud in South Africa using a proactive approach

Jean Damascene Mvunabandi, Bomi Nomlala

Currency Momentum: An Emerging Market Issue?

Maik Schober, Matthias Gehrke

Does ERM Sophistication Drive IPO Initial Performance in Emerging Market? Evidence from Malaysian Market Norliza Che-Yahya, Siti Sarah Alyasa-Gan, Rasidah Mohd-Rashid

Does IFRS 7 Disclosure Weaken Earnings Management?

Evidence from Indonesian Conventional Commercial Banks

Riyan Harbi Valdiansyah, Etty Murwaningsari, Sekar Mayangsari 